\title{
Differential subordination applications to a class of meromorphic multivalent functions associated with Mittag-Leffler function
}

\author{
Adela O. Mostafa ${ }^{1}$ and Ghada M. El-hawsh ${ }^{*}$
}

*Correspondence:
gma05@fayoum.edu.eg
2Department of Mathematics,
Faculty of Science, Fayoum
University, Fayoum 63514, Egypt
Full list of author information is
available at the end of the article

available at the end of the article

\begin{abstract}
In this paper, using the principal of differential subordination, we obtain some properties of certain class of $p$-valent meromorphic functions, which are defined by Mittag-Leffler function.
\end{abstract}

Keywords: Analytic function, Convex function, Meromorphic multivalent functions, Subordination and Mittag-Leffler function

2010 mathematics subject classification: $30 C 45,30 C 50,30 C 55$

\section{Introduction}

Denote by $\Sigma_{p, m}$ the class of analytic meromorphic multivalent functions of the form:

$$
f(z)=\frac{1}{z^{p}}+\sum_{k=m}^{\infty} a_{k} z^{k}(p \in \mathbb{N}=\{1,2, \ldots\} ; m>-p),
$$

where $\mathbb{U}^{*}=\{z \in \mathbb{C}$ and $0<|z|<1\}=\mathbb{U} \backslash\{0\}$. We note that Sigma $a_{p, 1-p}=\Sigma_{p}$.

For two functions $f(z)$ and $g(z)$, analytic in $\mathbb{U}, f(z)$ is subordinate to $g(z)(f(z) \prec g(z))$ in $\mathbb{U}$, if there exists a function $\omega(z)$, analytic in $\mathbb{U}$ with $\omega(0)=0$ and $|\omega(z)|<1, f(z)=$ $g(\omega(z))(z \in \mathbb{U})$ and if $g(z)$ is univalent in $\mathbb{U}$, then (see for details [1] and also [2])

$$
f(z) \prec g(z) \Longleftrightarrow f(0)=g(0) \text { and } f(\mathbb{U}) \subset g(\mathbb{U}) \text {. }
$$

The Hadamard product of $f(z)$ and $g(z)$ given by

$$
g(z)=\frac{1}{z^{p}}+\sum_{k=m}^{\infty} b_{k} z^{k}
$$

is defined by

$$
(f * g)(z)=\frac{1}{z^{p}}+\sum_{k=m}^{\infty} a_{k} b_{k} z^{k}=(g * f)(z)
$$

(c) The Author(s). 2019 Open Access This article is distributed under the terms of the Creative Commons Attribution 4.0 International License (http://creativecommons.org/licenses/by/4.0/), which permits unrestricted use, distribution, and reproduction in any medium, provided you give appropriate credit to the original author(s) and the source, provide a link to the Creative Commons license, and indicate if changes were made. 
The Mittag-Leffler function $E_{\alpha}(z)\left(z \in \mathbb{U}^{*}\right)$ ([3] and [4]) see also ([5, 6] and [7]) is defined by

$$
E_{\alpha}(z)=\sum_{k=0}^{\infty} \frac{1}{\Gamma(k \alpha+1)} z^{k}, \alpha \in \mathbb{C}, \Re(\alpha)>0 .
$$

For $\alpha, \beta, \gamma \in \mathbb{C}, \mathfrak{R}(\alpha)>0, \max \{0, \mathfrak{R}(c)-1\}$ and $\Re(c)>0$, Srivastava and Tomovski [8] generalized Mittag-Leffler function by the function

$$
E_{\alpha, \beta}^{\gamma, c}(z)=\sum_{k=0}^{\infty} \frac{(\gamma)_{k c}}{\Gamma(k \alpha+\beta) k !} z^{n k}
$$

and proved that it is an entire function in the complex $z$-plane, where

$$
(\gamma)_{\theta}=\frac{\Gamma(\gamma+\theta)}{\Gamma(\gamma)}\left\{\begin{array}{c}
1, \quad \theta=0 \\
\gamma(\gamma+1) \ldots(\gamma+\theta-1), \theta \neq 0
\end{array} .\right.
$$

Mostafa and Aouf [9] (see also [10]) used the function $E_{\alpha, \beta}^{\gamma, c}(z)$ and defined the meromorphic function

$$
\begin{aligned}
\mathcal{M}_{p, \alpha, \beta}^{\gamma, c}(z)= & z^{-p} \Gamma(\beta) E_{\alpha, \beta}^{\gamma, c}(z) \\
= & z^{-p}+\sum_{k=m}^{\infty} \frac{\Gamma(\beta) \Gamma[\gamma+(k+p) c]}{\Gamma(\gamma) \Gamma[\beta+(k+p) \alpha](k+p) !} z^{k}, \\
& (\Re(\alpha)=0 \text { when } \Re(c)=1 \text { with } \beta \neq 0),
\end{aligned}
$$

and for $f(z) \in \Sigma_{p, m}$, they defined the operator

$$
\begin{aligned}
\mathcal{H}_{p, \alpha, \beta}^{\gamma, c} f(z) & =\mathcal{M}_{p, \alpha, \beta}^{\gamma, c}(z) * f(z) \\
& =z^{-p}+\sum_{k=m}^{\infty} \frac{\Gamma(\beta) \Gamma[\gamma+(k+p) c]}{\Gamma(\gamma) \Gamma[\beta+(k+p) \alpha](k+p) !} a_{k} z^{k}
\end{aligned}
$$

From (5) it is easy to have

$$
c z\left(\mathcal{H}_{p, \alpha, \beta}^{\gamma, c} f(z)\right)^{\prime}=\gamma \mathcal{H}_{p, \alpha, \beta}^{\gamma+1, c} f(z)-(\gamma+p c) \mathcal{H}_{p, \alpha, \beta}^{\gamma, c} f(z)(c>0)
$$

and

$$
\alpha z\left(\mathcal{H}_{p, \alpha, \beta+1}^{\gamma, c} f(z)\right)^{\prime}=\beta \mathcal{H}_{p, \alpha, \beta}^{\gamma, c} f(z)-(p \alpha+\beta) \mathcal{H}_{p, \alpha, \beta+1}^{\gamma, c} f(z), \alpha \neq 0 .
$$

We note that:

(i) $\mathcal{H}_{p, 0, \beta}^{1,1} f(z)=f(z)$;

(ii) $\mathcal{H}_{p, 0, \beta}^{2,1} f(z)=(p+1) f(z)+z f^{\prime}(z)$;

(iii) $\mathcal{H}_{1,0, \beta}^{2,1} f(z)=2 f(z)+z f^{\prime}(z)$.

Using the operator $\mathcal{H}_{p, \alpha, \beta}^{\gamma, c} f(z)$, we have the following definition.

Definition 1. For fixed $A$ and $B(-1 \leq B<A \leq 1)$, we say that a function $f \in \Sigma_{p, m}$ is in the class $\Sigma_{p, m}^{\gamma, c}(\alpha, \beta ; A, B)$ if it satisfies

$$
-\frac{z^{p+1}\left(\mathcal{H}_{p, \alpha, \beta}^{\gamma, c} f(z)\right)^{\prime}}{p} \prec \frac{1+A z}{1+B z} .
$$


In view of the definition of differential subordination, (8) is equivalent to

$$
\left|\frac{z^{p+1}\left(\mathcal{H}_{p, \alpha, \beta}^{\gamma, c} f(z)\right)^{\prime}+p}{B z^{p+1}\left(\mathcal{H}_{p, \alpha, \beta}^{\gamma, c} f(z)\right)^{\prime}+p A}\right|<1 .
$$

We note that:

(i)

$$
\begin{aligned}
\Sigma_{p, 1}^{1,1}(0,1 ; A, B) & =\Sigma_{p}(A, B)\left(-1 \leq B<A \leq 1 ; \mathbb{U}^{*}\right) \\
& =\left\{f \in \Sigma_{p}:-\frac{z^{p+1} f^{\prime}(z)}{p} \prec \frac{1+A z}{1+B z}\right\},
\end{aligned}
$$

the class $\Sigma_{p}(A, B)$ was introduced and studied by Mogra [11].

(ii)

$$
\begin{aligned}
\Sigma_{p, m}^{\gamma, c}\left(\alpha, \beta ; 1-\frac{2 \eta}{p},-1\right) & =\Sigma_{p, m}^{\gamma, c}(\alpha, \beta, \eta)(0 \leq \eta<p) \\
& =\left\{f \in \Sigma_{p, m}: \Re\left\{-z^{p+1}\left(\mathcal{H}_{p, \alpha, \beta}^{\gamma, c} f(z)\right)^{\prime}\right\}>\eta\right\}
\end{aligned}
$$

\section{Preliminary results}

The following lemmas will be required in our investigation.

Lemma 1 [12]. Let h be a convex (univalent) function in $\mathbb{U}$ with $h(0)=1$. Also let

$$
\phi(z)=1+d_{p+m} z^{p+m}+d_{p+m+1} z^{p+m+1}+\ldots,
$$

be analytic in $\mathbb{U}$. If

$$
\phi(z)+\frac{z \phi^{\prime}(z)}{\tau} \prec h(z)(\Re(\tau) \geq 0 ; \tau \neq 0 ; z \in \mathbb{U}),
$$

then

$$
\phi(z) \prec \Psi(z)=\frac{\tau}{p+m} z^{-\frac{\tau}{p+m}} \int_{0}^{z} t^{\frac{\tau}{p+m}-1} h(t) d t .
$$

Lemma 2 [13]. Let $\mu$ be a positive measure on the unit interval $[0,1]$. Let $g(z, t)$ be a complex valued function defined on $\mathbb{U} \times[0,1]$ such that $g(., t)$ is analytic in $\mathbb{U}$ for each $t \in[0,1]$ and such that $g(z,$.$) is \mu$ integrable on $[0,1]$ for all $z \in \mathbb{U}$. In addition, suppose that $\Re\{g(z, t)\}>0, g(-r, t)$ is real and

$$
\Re\left\{\frac{1}{g(z, t)}\right\} \geq \frac{1}{g(-r, t)}(|z| \leq r<1 ; t \in[0,1]) .
$$

If the function $G$ is defined by

$$
G(z)=\int_{0}^{1} g(z, t) d \mu(t),
$$

then

$$
\mathfrak{R}\left\{\frac{1}{G(z)}\right\} \geq \frac{1}{G(-r)}(|z| \leq r<1) .
$$


Each of the identities (asserted by Lemma 2) is fairly well known (cf., e.g., [[8], ch. 14]).

Lemma 3 [14]. For real or complex numbers $a, b$, and $c(c \neq 0,-1,-2, \ldots)$

$$
\begin{aligned}
& \int_{0}^{1} t^{b-1}(1-t)^{c-b-1}(1-t z)^{-a} d t=\frac{\Gamma(b) \Gamma(c-b)}{\Gamma(c)} F_{1}(a, b ; c ; z)(\Re(c)<\Re(b)>0) ; \\
& { }_{2} F_{1}(a, b ; c ; z)=(1-z)_{2}^{-a} F_{1}\left(a, c-b ; c ; \frac{z}{z-1}\right)(z \neq 1)
\end{aligned}
$$

and

$$
{ }_{2} F_{1}(a, b ; c ; z)={ }_{2} F_{1}(b, a ; c ; z) .
$$

Lemma 4 [15]. Let $\Phi$ be analytic in $\mathbb{U}$ with

$$
\Phi(0)=1 \text { and } \Re\{\Phi(z)\}>\frac{1}{2} .
$$

Then, for any function $F$, analytic in $\mathbb{U},(\Phi * F)(\mathbb{U})$ is contained in the convex hull of $F(\mathbb{U})$.

We used the technique used by ([16-18] and [19]).

\section{Main inclusion relationships}

Unless otherwise mentioned, we assume throughout this paper that $-1 \leq B<A \leq$ $1, \alpha, \beta, \gamma \in \mathbb{C}, \Re(\alpha)>0, \max \{0, \Re(c)-1\}, \Re(c)>0, \delta>0, f(z)$ given by $(1)$ and $z \in \mathbb{U}^{*}$.

Theorem 1 Let $\gamma \neq 0$ and $f(z)$ satisfy:

$$
-\frac{(1-\delta) z^{p+1}\left(\mathcal{H}_{p, \alpha, \beta}^{\gamma, c} f(z)\right)^{\prime}+\delta z^{p+1}\left(\mathcal{H}_{p, \alpha, \beta}^{\gamma+1, c} f(z)\right)^{\prime}}{p} \prec \frac{1+A z}{1+B z},
$$

then

$$
-\frac{z^{p+1}\left(\mathcal{H}_{p, \alpha, \beta}^{\gamma, c} f(z)\right)^{\prime}}{p} \prec \Psi(z) \prec \frac{1+A z}{1+B z},
$$

where

$$
\Psi(z)=\left\{\begin{array}{cl}
\frac{A}{B}+\left(1-\frac{A}{B}\right)(1+B z)^{-1} F_{1}\left(1,1 ; \frac{\gamma}{\delta c(p+m)}+1 ; \frac{B z}{1+B z}\right), & B \neq 0 \\
1+\frac{\gamma}{\gamma+\delta c(p+m)} A z, & B=0 .
\end{array}\right.
$$

is the best dominant of (17). Furthermore,

$$
\Re\left\{-\frac{z^{p+1}\left(\mathcal{H}_{p, \alpha, \beta}^{\gamma, c} f(z)\right)^{\prime}}{p}\right\}>\rho(0 \leq \rho<1),
$$

where

$$
\rho=\left\{\begin{array}{cl}
\frac{A}{B}+\left(1-\frac{A}{B}\right)(1-B){ }_{2}^{-1} F_{1}\left(1,1 ; \frac{\gamma}{\delta c(p+m)}+1 ; \frac{B}{B-1}\right), & B \neq 0 \\
1-\frac{\gamma}{\gamma+\delta c(p+m)} A, & B=0 .
\end{array}\right.
$$

The result is the best possible. 
Proof Let

$$
\phi(z)=-\frac{z^{p+1}\left(\mathcal{H}_{p, \alpha, \beta}^{\gamma, c} f(z)\right)^{\prime}}{p},
$$

where $\phi$ is given by (11). Differentiating (21) and using (6), we get

$$
-\frac{(1-\delta) z^{p+1}\left(\mathcal{H}_{p, \alpha, \beta}^{\gamma, c} f(z)\right)^{\prime}+\delta z^{p+1}\left(\mathcal{H}_{p, \alpha, \beta}^{\gamma+1, c} f(z)\right)^{\prime}}{p}=\phi(z)+\frac{\delta c z \phi^{\prime}(z)}{\gamma} \prec \frac{1+A z}{1+B z} .
$$

Now, by using Lemma 1 for $\tau=\frac{\gamma}{\delta c}$, we get

$$
\begin{aligned}
-\frac{z^{p+1}\left(\mathcal{H}_{p, \alpha, \beta}^{\gamma, c} f(z)\right)^{\prime}}{p} & \prec \Psi(z)=\frac{\gamma}{\delta c(p+m)} z^{-\frac{\gamma}{\delta c(p+m)} \int_{0}^{z} t^{\frac{\gamma}{\delta c(p+m)}}-1}\left(\frac{1+A t}{1+B t}\right) d t \\
& =\left\{\begin{array}{cc}
\frac{A}{B}+\left(1-\frac{A}{B}\right)(1+B z)_{2}^{-1} F_{1}\left(1,1 ; \frac{\gamma}{\delta c(p+m)}+1 ; \frac{B z}{1+B z}\right), & B \neq 0 \\
1+\frac{\gamma}{\gamma+\delta c(p+m)} A z, & B=0 .
\end{array}\right.
\end{aligned}
$$

This proves (17) of Theorem 1. In order to prove (20), we need to show that

$$
\inf _{|z|<1}\{\Re(\Psi(z))\}=\Psi(-1) .
$$

We have

$$
\Re\left\{\frac{1+A z}{1+B z}\right\} \geq \frac{1-A r}{1-B r}(|z| \leq r<1) .
$$

Putting

$$
G(z, \zeta)=\frac{1+A \zeta z}{1+B \zeta z} \text { and } d v(\zeta)=\frac{\gamma}{\delta c(p+m)} \zeta^{\frac{\gamma}{c(p+m)}-1} d \zeta(0 \leq \zeta \leq 1),
$$

which is a positive measure on $[0,1]$, we obtain

$$
\Psi(z)=\int_{0}^{1} G(z, \zeta) d v(\zeta) .
$$

Then

$$
\Re(\Psi(z)) \geq \int_{0}^{1} \frac{1-A \zeta r}{1-B \zeta r} d v(\zeta)=\Psi(-r)(|z| \leq r<1) .
$$

Assuming $r \rightarrow 1^{-}$in the above inequality, we obtain (22). The result in (19) is the best possible and $\Psi$ is the best dominant of (17). This completes the proof of Theorem 1.

Theorem $2 \operatorname{Let} f(z) \in \Sigma_{p, m}^{\gamma, c}(\alpha, \beta, \eta)(0 \leq \eta<p)$, then

$$
\Re\left\{-z^{p+1}\left[(1-\delta)\left(\mathcal{H}_{p, \alpha, \beta}^{\gamma, c} f(z)\right)^{\prime}+\delta\left(\mathcal{H}_{p, \alpha, \beta}^{\gamma+1, c} f(z)\right)^{\prime}\right]\right\}>\eta(|z|<R),
$$

where

$$
R=\left\{\frac{\sqrt{c^{2} \delta^{2}(p+m)^{2}+\gamma^{2}}-c \delta(p+m)}{\gamma}\right\}^{\frac{1}{p+m}} .
$$

The result is the best possible. 
Proof Since $f(z) \in \Sigma_{p, m}^{\gamma, c}(\alpha, \beta, \eta)$, let

$$
-z^{p+1}\left(\mathcal{H}_{p, \alpha, \beta}^{\gamma, c} f(z)\right)^{\prime}=\eta+(p-\eta) u(z)
$$

where $u(z)$ in the form (11) and $\Re\{u(z)\}>0$. Differentiating (25) and using (6), we get

$$
-\frac{z^{p+1}\left[(1-\delta)\left(\mathcal{H}_{p, \alpha, \beta}^{\gamma, c} f(z)\right)^{\prime}+\delta\left(\mathcal{H}_{p, \alpha, \beta}^{\gamma+1, c} f(z)\right)^{\prime}\right]+\eta}{p-\eta}=u(z)+\frac{c \delta z u^{\prime}(z)}{\gamma} .
$$

Applying the following estimate [20],

$$
\frac{\left|z u^{\prime}(z)\right|}{\Re\{u(z)\}} \leq \frac{2(p+m) r^{p+m}}{1-r^{2(p+m)}}(|z|=r<1) ；
$$

in (26), we get

$$
\begin{aligned}
& \Re\left\{-\frac{z^{p+1}\left[(1-\delta)\left(\mathcal{H}_{p, \alpha, \beta}^{\gamma, c} f(z)\right)^{\prime}+\delta\left(\mathcal{H}_{p, \alpha, \beta}^{\gamma+1, c} f(z)\right)^{\prime}\right]+\eta}{p-\eta}\right\} \\
\geq & \Re(u(z))\left(1-\frac{2 c \delta(p+m) r^{p+m}}{\gamma\left(1-r^{2(p+m)}\right)}\right) .
\end{aligned}
$$

It is easily seen that the right-hand side of (27) is positive, if $r<R$, where $R$ is given by (24). In order to show that the bound $R$ is the best possible, we consider the function $f \in \Sigma_{p, m}$ defined by

$$
-z^{p+1}\left(\mathcal{H}_{p, \alpha, \beta}^{\gamma, c} f(z)\right)^{\prime}=\eta+(p-\eta) \frac{1+z^{p+m}}{1-z^{p+m}} .
$$

Noting that

$$
\begin{aligned}
- & \frac{z^{p+1}\left[(1-\delta)\left(\mathcal{H}_{p, \alpha, \beta}^{\gamma, c} f(z)\right)^{\prime}+\delta\left(\mathcal{H}_{p, \alpha, \beta}^{\gamma+1, c} f(z)\right)^{\prime}\right]+\eta}{p-\eta} \\
= & \frac{\gamma\left(1-z^{2(p+m)}\right)+2 c \delta(p+m) z^{p+m}}{\gamma\left(1-z^{p+m}\right)^{2}}=0,
\end{aligned}
$$

for

$$
z=R \Re\left(\frac{i \pi}{p+m}\right) .
$$

This completes the proof of Theorem 2 .

Putting $\delta=1$ in Theorem 2, we obtain the following result.

Corollary 1 Iff $(z) \in \Sigma_{p, m}^{\gamma, c}(\alpha, \beta, \eta)(0 \leq \eta<p)$, then $f(z) \in \Sigma_{p, m}^{\gamma+1, c}(\alpha, \beta, \eta)$ for $|z|<R^{*}$, where

$$
R^{*}=\left\{\frac{\sqrt{c^{2}(p+m)^{2}+\gamma^{2}}-c(p+m)}{\gamma}\right\}^{\frac{1}{p+m}} .
$$

The result is the best possible. 
Theorem 3 If the function $f(z) \in \Sigma_{p, m}$ satisfies

$$
z^{p}\left[(1-\delta) \mathcal{H}_{p, \alpha, \beta}^{\gamma, c} f(z)+\delta \mathcal{H}_{p, \alpha, \beta}^{\gamma+1, c} f(z)\right] \prec \frac{1+A z}{1+B z},
$$

then

$$
z^{p} \mathcal{H}_{p, \alpha, \beta}^{\gamma, c} f(z) \prec \Psi_{1}(z) \prec \frac{1+A z}{1+B z}
$$

and

$$
\Re\left\{z^{p} \mathcal{H}_{p, \alpha, \beta}^{\gamma, c} f(z)\right\}>\rho,
$$

where $\Psi_{1}(z)$ is in the form (18) and $\rho$ given by (20). The result is the best possible.

Proof The proof follows by taking the same lines as in the proof of Theorem 1 and taking $\phi(z)=z^{p} \mathcal{H}_{p, \alpha, \beta}^{\gamma, c} f(z)$ in (21).

For the function $f(z)$ in the class $\Sigma_{p, m}$, Kumar and Shukla [21] defined the integral operator $\digamma_{\mu, p}: \Sigma_{p, m} \rightarrow \Sigma_{p, m}$ as follows:

$$
\begin{aligned}
\digamma_{\mu, p}(f)(z) & =\frac{\mu}{z^{\mu+p}} \int_{0}^{z} t^{\mu+p-1} f(t) d t \\
& =z^{-p}+\sum_{k=m}^{\infty} \frac{\mu}{k+p+\mu} a_{k} z^{k}(\mu>0) .
\end{aligned}
$$

From (28), we get

$$
z\left(\mathcal{H}_{p, \alpha, \beta}^{\gamma, c} \digamma_{\mu, p}(f)(z)\right)^{\prime}=\mu \mathcal{H}_{p, \alpha, \beta}^{\gamma, c} f(z)-(\mu+p) \mathcal{H}_{p, \alpha, \beta}^{\gamma, c} \digamma_{\mu, p}(f)(z)
$$

Theorem 4 Let the function $f(z)$ given by (1) be in the class $\Sigma_{p, m}^{\gamma, c}(\alpha, \beta ; A, B)$ and $\digamma_{\mu, p}(f)(z)$ defined by (28). Then

$$
-\frac{z^{p+1}\left(\mathcal{H}_{p, \alpha, \beta}^{\gamma, c} \digamma_{\mu, p}(f)(z)\right)^{\prime}}{p} \prec \Theta(z) \prec \frac{1+A z}{1+B z},
$$

where

$$
\Theta(z)=\left\{\begin{array}{cl}
\frac{A}{B}+\left(1-\frac{A}{B}\right)(1+B z)_{2}^{-1} F_{1}\left(1,1 ; \frac{\mu}{(p+m)}+1 ; \frac{B z}{1+B z}\right), & B \neq 0 \\
1+\frac{\mu}{\mu+p+m} A z, & B=0 .
\end{array}\right.
$$

is the best dominant of (31). Furthermore,

$$
\Re\left\{-\frac{z^{p+1}\left(\mathcal{H}_{p, \alpha, \beta}^{\gamma, c} \digamma_{\mu, p}(f)(z)\right)^{\prime}}{p}\right\}>\sigma(0 \leq \sigma<1),
$$

where

$$
\sigma=\left\{\begin{array}{cl}
\frac{A}{B}+\left(1-\frac{A}{B}\right)(1-B)^{-1} F_{1}\left(1,1 ; \frac{\mu}{(p+m)}+1 ; \frac{B}{B-1}\right), & B \neq 0 \\
1-\frac{\mu}{\mu+p+m} A, & B=0 .
\end{array}\right.
$$

The result is the best possible. 
Proof Let

$$
L(z)=-\frac{z^{p+1}\left(\mathcal{H}_{p, \alpha, \beta}^{\gamma, c} \digamma_{\mu, p}(f)(z)\right)^{\prime}}{p},
$$

where $L$ in the form (11). Differentiating (34) and using (29), we get

$$
-\frac{z^{p+1}\left(\mathcal{H}_{p, \alpha, \beta}^{\gamma, c} f(z)\right)^{\prime}}{p}=L(z)+\frac{z}{\mu} L^{\prime}(z) \prec \frac{1+A z}{1+B z} .
$$

Now the remaining part of Theorem 4 follows by using the technique used in proving Theorem 1.

Theorem 5 Let the function $\digamma_{\mu, p}(f)(z)$ defined by (28) satisfy:

$$
z^{p}\left[(1-\delta) \mathcal{H}_{p, \alpha, \beta}^{\gamma, c} \digamma_{\mu, p}(f)(z)+\delta \mathcal{H}_{p, \alpha, \beta}^{\gamma, c} f(z)\right] \prec \frac{1+A z}{1+B z},
$$

then

$$
\Re\left\{z^{p} \mathcal{H}_{p, \alpha, \beta}^{\gamma, c} \digamma_{\mu, p}(f)(z)\right\}>\theta,
$$

where

$$
\theta=\left\{\begin{array}{cr}
\frac{A}{B}+\left(1-\frac{A}{B}\right)(1-B)_{2}^{-1} F_{1}\left(1,1 ; \frac{\mu}{\delta(p+m)}+1 ; \frac{B}{B-1}\right), & B \neq 0 \\
1-\frac{\mu}{\mu+\delta(p+m)} A, & B=0 .
\end{array}\right.
$$

The result is the best possible.

\section{Proof Let}

$$
K(z)=z^{p} \mathcal{H}_{p, \alpha, \beta}^{\gamma, c} \digamma_{\mu, p}(f)(z),
$$

where $K$ in the form (11). Differentiating (37) and using (29) and (35), we get

$$
K(z)+\frac{\delta z}{\mu} K^{\prime}(z) \prec \frac{1+A z}{1+B z} .
$$

Now the remaining part of Theorem 5 follows by using the technique used in proving Theorem 1.

Theorem 6 Let the function $f(z) \in \Sigma_{p, m}$ satisfy:

$$
-\frac{z^{p+1}\left[(1-\delta)\left(\mathcal{H}_{p, \alpha, \beta}^{\gamma, c} \digamma_{\mu, p}(f)(z)\right)^{\prime}+\delta\left(\mathcal{H}_{p, \alpha, \beta}^{\gamma, c} f(z)\right)^{\prime}\right]}{p} \prec \frac{1+A z}{1+B z},
$$

then

$$
\Re\left\{-\frac{z^{p+1}\left(\mathcal{H}_{p, \alpha, \beta}^{\gamma, c} \digamma_{\mu, p}(f)(z)\right)^{\prime}}{p}\right\}>\theta,
$$

where $\digamma_{\mu, p}(f)(z)$ is given by (28) and $\theta$ is given as in Theorem 5. The result is the best possible.

Proof The proof follows by taking the same lines as in Theorem 5 . 
Theorem 7 Let $f(z)$ be in the class $\Sigma_{p, m}$. Also, let $g(z) \in \Sigma_{p, m}$ satisfy:

$$
\Re\left\{z^{p} \mathcal{H}_{p, \alpha, \beta}^{\gamma, c} g(z)\right\}>0 .
$$

If

$$
\left|\frac{\mathcal{H}_{p, \alpha, \beta}^{\gamma, c} f(z)}{\mathcal{H}_{p, \alpha, \beta}^{\gamma, c} g(z)}-1\right|<1
$$

then

$$
\Re\left\{-\frac{z\left(\mathcal{H}_{p, \alpha, \beta}^{\gamma, c} f(z)\right)^{\prime}}{\mathcal{H}_{p, \alpha, \beta}^{\gamma, c} f(z)}\right\}>0\left(|z|<R_{0}\right),
$$

where

$$
R_{0}=\frac{\sqrt{9(p+m)^{2}+4 p(2 p+m)}-3(p+m)}{2(2 p+m)} .
$$

Proof Let

$$
\phi(z)=\frac{\mathcal{H}_{p, \alpha, \beta}^{\gamma, c} f(z)}{\mathcal{H}_{p, \alpha, \beta}^{\gamma, c} g(z)}-1=e_{p+m} z^{p+m}+e_{p+m+1} z^{p+m+1}+\ldots,
$$

we note that $\phi$ is analytic in $\mathbb{U}$, with $\phi(0)=0$ and $|\phi(z)| \leq|z|^{p+m}$. Then, by applying the familiar Schwarz Lemma [22], we have $\phi(z)=z^{p+m} \Psi(z)$ is analytic in $\mathbb{U}$ and $|\Psi(z)| \leq 1$ $(z \in \mathbb{U})$. Therefore, (40) leads to

$$
\mathcal{H}_{p, \alpha, \beta}^{\gamma, c} f(z)=\mathcal{H}_{p, \alpha, \beta}^{\gamma, c} g(z)\left(z^{p+m} \Psi(z)+1\right) .
$$

Differentiating (41), we obtain

$$
\frac{z\left(\mathcal{H}_{p, \alpha, \beta}^{\gamma, c} f(z)\right)^{\prime}}{\mathcal{H}_{p, \alpha, \beta}^{\gamma, c} f(z)}=\frac{z\left(\mathcal{H}_{p, \alpha, \beta}^{\gamma, c} g(z)\right)^{\prime}}{\mathcal{H}_{p, \alpha, \beta}^{\gamma, c} g(z)}+\frac{z^{p+m}\left[(p+m) \Psi(z)+z \Psi^{\prime}(z)\right]}{1+z^{p+m} \Psi(z)} .
$$

Letting $\chi(z)=z^{p} \mathcal{H}_{p, \alpha, \beta}^{\gamma, c} g(z)$, we see that the function $\chi$ is of the form (11) and is analytic in $\mathbb{U}, \mathfrak{R}\{\chi(z)\}>0$ and

$$
\frac{z\left(\mathcal{H}_{p, \alpha, \beta}^{\gamma, c} g(z)\right)^{\prime}}{\mathcal{H}_{p, \alpha, \beta}^{\gamma, c} g(z)}=\frac{z \chi^{\prime}(z)}{\chi(z)}-p,
$$

so, we find from (42) that

$$
\Re\left\{-\frac{z\left(\mathcal{H}_{p, \alpha, \beta}^{\gamma, c} f(z)\right)^{\prime}}{\mathcal{H}_{p, \alpha, \beta}^{\gamma, c} f(z)}\right\} \geq p-\left|\frac{z \chi^{\prime}(z)}{\chi(z)}\right|-\left|\frac{z^{p+m}\left[(p+m) \Psi(z)+z \Psi^{\prime}(z)\right]}{1+z^{p+m} \Psi(z)}\right| .
$$

Using the following known estimates [23] (see also [20]),

$$
\left|\frac{\chi^{\prime}(z)}{\chi(z)}\right| \leq \frac{2(p+m) r^{p+m-1}}{1-r^{2(p+m)}} \text { and }\left|\frac{(p+m) \Psi(z)+z \Psi^{\prime}(z)}{1+z^{p+m} \Psi(z)}\right| \leq \frac{p+m}{1-r^{p+m}}(|z|=r<1),
$$

in (43), we have

$$
\Re\left\{-\frac{z\left(\mathcal{H}_{p, \alpha, \beta}^{\gamma, c} f(z)\right)^{\prime}}{\mathcal{H}_{p, \alpha, \beta}^{\gamma, c} f(z)}\right\} \geq \frac{p-3(p+m) r^{p+m}-(2 p+m) r^{2(p+m)}}{1-r^{2(p+m)}},
$$

which is certainly positive, provided that $r<R_{0}, R_{0}$ given by (39). 
Theorem 8 Let the function $f(z) \in \Sigma_{p, m}$ satisfy:

$$
z^{p}\left[(1-\delta) \mathcal{H}_{p, \alpha, \beta}^{\gamma, c} f(z)+\delta \mathcal{H}_{p, \alpha, \beta}^{\gamma+1, c} f(z)\right] \prec \frac{1+A z}{1+B z},
$$

then

$$
\Re\left\{\left(z^{p} \mathcal{H}_{p, \alpha, \beta}^{\gamma, c} f(z)\right)^{\frac{1}{q}}\right\}>\epsilon^{\frac{1}{q}}(q \in \mathbb{N}),
$$

where $\epsilon$ in the form (20). The result is the best possible.

Proof Let

$$
\phi(z)=z^{p} \mathcal{H}_{p, \alpha, \beta}^{\gamma, c} f(z),
$$

where $\phi$ in the form (11). Differentiating (44) and using (6), we have

$$
z^{p}\left[(1-\delta) \mathcal{H}_{p, \alpha, \beta}^{\gamma, c} f(z)+\delta \mathcal{H}_{p, \alpha, \beta}^{\gamma+1, c} f(z)\right]=\phi(z)+\frac{\delta c z \phi^{\prime}(z)}{\gamma} \prec \frac{1+A z}{1+B z} .
$$

Now the remaining part of Theorem 8 follows by using the technique used in proving Theorem 1, and using the inequality:

$$
\Re\left(w^{\frac{1}{q}}\right) \geq(\Re(w))^{\frac{1}{q}}(\Re(w)>0 ; q \in \mathbb{N}),
$$

we have the result asserted by Theorem 8 .

Theorem 9 Let the function $f(z) \in \Sigma_{p, m}^{\gamma, c}(\alpha, \beta ; A, B)$ and let $g(z) \in \Sigma_{p, m}$ satisfy:

$$
\Re\left(z^{p} g(z)\right)>\frac{1}{2} \text {. }
$$

Then,

$$
(f * g)(z) \in \Sigma_{p, m}^{\gamma, c}(\alpha, \beta ; A, B) .
$$

Proof We have

$$
-\frac{z^{p+1}\left(\mathcal{H}_{p, \alpha, \beta}^{\gamma, c}(f * g)(z)\right)^{\prime}}{p}=-\frac{z^{p+1}\left(\mathcal{H}_{p, \alpha, \beta}^{\gamma, c} f(z)\right)^{\prime}}{p} * z^{p} g(z) .
$$

Since

$$
\Re\left(z^{p} g(z)\right)>\frac{1}{2}
$$

and $\frac{1+A z}{1+B z}$ is convex in $\mathbb{U}$, it follows from (8) and Lemma 4 that $(f * g)(z) \in$ $\Sigma_{p, m}^{\gamma, c}(\alpha, \beta ; A, B)$, which completes the proof of Theorem 9.

Remark 1 For different value of $\gamma, c, \alpha, \beta$, and $p$ in the above results, we obtain results corresponding to the functions given in the introduction.

\section{Acknowledgement}

(i) The authors wish to thank Prof. Dr. M. K. Aouf for his kindly encouragement and help in the preparation of this paper. (ii) The authors are thankful to the referees for helpful suggestions.

\section{Authors' contributions}

Both authors read and approved the final manuscript.

Funding

The authors declare that they had no funding. 


\section{Competing interests}

The authors declare that they have no competing interests.

\section{Author details}

${ }^{1}$ Department of Mathematics, Faculty of Science, Mansoura University, Mansoura 35516, Egypt. ${ }^{2}$ Department of Mathematics, Faculty of Science, Fayoum University, Fayoum 63514, Egypt.

Received: 24 April 2019 Accepted: 15 July 2019

Published online: 28 August 2019

\section{References}

1. Bulboaca, T.: Differential Subordinations and Superordinations, Recent Results, Cluj-Napoca (2005)

2. Miller, S. S., Mocanu, P. T.: Differential Subordinations: Theory and Applications, Series on Monographs and Textbooks in Pure and Applied Mathematics, vol. 225. Marcel Dekker, New York and Basel (2000)

3. Mittag-Leffler, G. M.: Sur la nouvelle function. C.R. Acad. Sci., Paris. 137, 554-558 (1903)

4. Mittag-Leffler, G. M.: Sur la representation analytique d'une function monogene (cinquieme note). Acta Math. 29 101-181 (1905)

5. Kiryakova, V. S.: Multiple (multi-index) Mittag-Leffler functions and relations to generalized fractional calculus. Higher transcendental functions and their applications. J. Comput. Appl. Math. 118(1-2), 241-259 (2000)

6. Kiryakova, V:: The multi-index Mittag-Leffler functions as an important class of special functions of fractional calculus, Comput. Math. Appl. 59(5), 1885-1895 (2010)

7. Kiryakova, V.: Criteria for univalence of the Dziok-Srivastava and the Srivastava-Wright operators in the class A, Appl. Math. Comput. 218(3), 883-892 (2011)

8. Srivastava, H. M., Tomovski, Z:: Fractional calculus with an integral operator containing a generalized Mittag-Leffler function in the kernal, Appl. Math. Comp. 211, 198-210 (2009)

9. Mostafa, A. O., Aouf, M. K.: Some inequalities for meromorphic multivalent functions associated with Mittag-Leffler function. J. Open Probl. Complex Anal. 10(1), 1-8 (2018)

10. Aouf, M. K., Seoudy, T. M.: Some families of meromorphic $p$-valent functions involving a new operator defined by generalized Mittag-Leffler function. J. Egy. Math. Soc. 26(3), 406-411 (2018)

11. Mogra, M. L.: Meromorphic multivalent functions with positive coefficients. I and II. Math. Japon. 35, 1-11 and 1089-1098 (1990)

12. Hallenbeck, D. Z., Ruscheweyh, St.: Subordination by convex functions. Proc. Amer. Math. Soc. 52, 191-195 (1975)

13. Willken, D. R., Feng, J.: A remark on convex and starlike functions. J. London. Math. Soc. 21(2), 287-290 (1980)

14. Whittaker, E. T., Watson, G. N.: A Course of Modern Analysis: an Introduction to the General Theory of Infinite Processes and of Analytic Functions: With an Account of the Principal Transcendental Functions, Fourth ed. Cambridge University Press, Cambridge (1927)

15. Singh, R., Singh, S.: Convolution properties of a class of starlike functions. Proc. Amer. Math. Soc. 106, 145-152 (1989)

16. Aouf, M. K: Applications of differential subordinations to certain subclasses of meromorphically multivalent functions associated with generalized hypergeometric function, Indian. J. Math. 52(2), 357-380 (2010)

17. EL-Ashwah, R. M., Aouf, M. K., Bulboaca, T.: Differential subordinations for classes of meromorphic $p$-valent functions defined by multiplier transformations. Bull. Aust. Math. Soc. 83, 353-368 (2011)

18. Mostafa, A. O.: Applications of differential subordination to certain subclasses of $p$-valent meromorphic functions involving a certain operators. Math. Comput. Modelling. 54, 1486-1498 (2011)

19. Seoudy, T. M., Aouf, M. K.: Some applications of differential subordination to certain subclasses of $p$-valent meromorphic functions involving convolution, Acta Univ. Sapientiae, Math. 6(1), 46-60 (2014)

20. MacGregor, T. H.: Radius of univalence of certain analytic functions. Proc. Amer. Math. Soc. 14, 514-520 (1963)

21. Kumar, V., Shukla, S. L.: Certain integrals for classes of $p$-valent meromorphic functions. Bull. Austral. Math. Soc. 25, 85-97 (1982)

22. Nehari, Z:: Conformal Mapping. McGraw-Hill, New York (1952)

23. Patel, J.: Radii of $\gamma$-spirallikeness of certain analytic functions. J. Math. Phys. Sci. 27, 321-334 (1993)

\section{Publisher's Note}

Springer Nature remains neutral with regard to jurisdictional claims in published maps and institutional affiliations. 